\title{
Equitability in Healthcare Resource Allocation Concerning Substance Users: An Argument Against Lower Priority Setting
}

\author{
La Shun L. Carroll \\ Abstract
}

\begin{abstract}
Organ transplantation centers set criteria for candidate qualification, which has led to disparate healthcare resource allocation practices affecting those with a substance use history. These individuals are denied organ transplants by committees and healthcare providers who assign them lower priority status. The lower priority argument claims that healthcare resources should not be provided equally to individuals failing to share responsibility for not doing enough in addressing the diseases associated with substance use. The purpose of this work is to explore the interrelatedness between the ethics of a merit-based system of moral responsibility and lower priority setting involved in healthcare resource allocation pertaining to those with substance use histories. An integral approach to the argument against the lower prioritists with a focus on the relationship between different organ allocation practices affecting substance users and the justification for resource allocation practices in healthcare and transplant committees. Lower priority setting is challenged, and an argument offered in which substance users are assigned higher priority when relying on "doing enough" in a merit-based system of moral responsibility. It is determined that one cannot substantiate assigning a lower priority status since a lack of success in rehab does not imply a lack of effort. Additionally, neither to confirmatory behavior, nor to non-conforming behavior may freedom be justifiably ascribed in a merit-based system of responsibility because freedom to choose can neither be established a priori, nor a posteriori with respect to meritorious behavior.
\end{abstract}

Keywords: Organ Transplantation, Ethics, Healthcare Resource Allocation, Lower Priority, Substance Use, Merit

La Shun L. Carroll is an Ed.M. graduate student at the University at Buffalo Graduate School of Education. He received his Doctorate, Cum Laude, from the University at Buffalo School of Dental Medicine, and a B.A., Magna Cum Laude, in Philosophy from Baruch College. His publications include "Theoretical Biomimetics: A biological design-driven concept for creative problem-solving as applied to the optimal sequencing of active learning techniques in educational theory" in Multidisciplinary Journal for Education, Social and Technological Sciences (October 2017), and "A Comprehensive Definition of Technology from an Ethological Perspective" (MDPI, 2017). Research interests include metaphysics, logic, science, technology, and education. Dr. Carroll was an Adjunct Professor at Saint Michael's College.

Author Contact:

La Shun L. Carroll, DDS, Ed.M.

Academic Email: lcarroll@buffalo.edu

Web: www.lashunlaruecarroll.com

ResearcherID: $\quad$ C-1838-2016

ORCID: http://orcid.org/0000-0003-4132-6392 


\section{Equitability in Healthcare Resource Allocation Concerning Substance Users: An Argument Against Lower Priority Setting}

Each day, approximately one person dies every hour as a result of the inability of vital organs supplies to meet the patient demand for healthcare resources in the United States (ATF, 2008). The ongoing debate concerning healthcare resources available for allocation centers around assessment procedures that would aid in the prioritization of potential candidates an efficiently and equitably (Neuberger, 1998). Nevertheless, as noted in Neuberger (1998), an agreement on how to directly prioritize patients according to an assessment protocol has proven to be a lot harder than expected. The reason for the difficulty relates to the manner in which to determine criteria that should be used for prioritization. This article will explore the justification for the author's contention that any criteria relied on for prioritization ought to take into account more than just a candidate's health status and his or her need for a particular resource.

\section{Frameworks Influence Criteria Selection}

Ethics is at the core of decision-making concerning the allocation of transplant resources. Given the nature of the transplantation, ethical theories may focus on either the donor, the recipient, the product being transplanted or the process of deciding to whom it should be allocated. Additionally, the motivation for the decisions made (e.g., why people donate and why boards choose particular people over others) or the consequences of the process (e.g., good, who benefits, or worthiness) will likely guide prioritization efforts. Because of these aspects, it would appear as though both deontological and consequential become the primary contention in selecting criteria in order to prioritize candidates in the author's opinion.

\section{Frameworks Influence Criteria Selection}

Briefly, deontological positions hold that there exists a "duty" in either procurement of things real or abstract or in committing particular actions based on the merit of their intrinsic value. Moreover, such intrinsic value ought to suffice in persuading one to act (Alexander \& Moore 2016). Conversely, when the merit behind procurement or committal is not perceived as being intrinsically situated within the context of the act or thing itself, but is, or ought to be, based on results or consequences of such, an individual may be said to espouse a consequentialist viewpoint according to Alexander \& Moore (2016).

There can be many different manifestations of either ethical position, as well. For instance, focusing on the "good" as a result, why wouldn't consequentialists aim to maximize it? Well, some do aim to do so, but it may come at a steep cost for those who 
do not benefit. Although the end is noble in appearance, the cost negates the value in some instances; the implication is that how that good is achieved is not a concern to the extent that intentional killing or otherwise violating individuals may be permissible to bring it about. This variety of consequentialism view may be referred to as utilitarianism and as you would imagine, is fraught with difficulties.

For example, how they value outcomes of the procedure and which outcomes of transplantation are determined good may allow for the ends to justify whatever means seen as necessary. Although this consequentialist manifestation seems to be farsighted with "good" in mind because they are guided mostly by the ends or outcomes (i.e., products) the individuals miss the forest for the trees.

The overall all picture or goal would be beneficence, yet if a group of people is to reap the greatest good, how can the most good of the group occur if one member has to be sacrificed? Surely, the groups sacrificial lamb effectively gets executed and excommunicated. Nevertheless, the interesting question is whether the decision to sacrifice the individual is made before or after the group members sacrifice the person and violate their rights.

Firstly, my understanding of group does not gerrymander the notion. In other words, conceptually, while a group A, for instance, may comprise a particular number of people at any moment in time-space, any alterations occurring in the number I contend renders this group no longer the same group A; it would be Group $\sim \mathrm{A}$. The members of a group characterize the group as though they were its identity, much like what imparts identity for the construct of the number 5 . To claim the group before and after is the same would be analogous to claiming $5=\sim 5$, which is absurd. Either sacrificing 1 member from 5 , or 3 members from 5, after the sacrifice the group cannot be the same group 5 because of an absence of its identity. In other words, the group after the sacrifice lost what characterized its group and therefore must be a different group. Furthermore, the new group comprised of remaining members were the ones benefitting; so when all 5 were in the original group A, if consequentialist said the greatest good for the group, he or she could not have included the one nominated for sacrifice among the group benefitting. So, what can one infer about the dynamic of the group beforehand?

Based on the previous information, not only did group A have a different identity than A after sacrificing someone resulting in different groups, but beforehand when the majority decided to sacrifice that one member. Regardless of the criteria used, since not among those benefiting, once the decision was made the group had already become a different unit psychologically. Precisely when did the change occur is the crucial question? 
If after sacrificing the group became $\sim \mathrm{A}$, then before sacrificing but after decisionmaking it can be argued that the group had already effectively transformed into $\sim \mathrm{A}$. The reason is that the one being sacrificed is not benefiting so he or she could not be considered among group A. In fact, even before act of choosing to sacrifice that unfortunate member, the group dynamic had to have changed; that change occurred in mindset the moment when greatest good for the greatest number of people meant excluding that one member benefiting. In effect, the consideration of greatest good in consequentialist frameworks may be viewed as exclusionary and discriminatory. To discriminate is to recognize distinction, according to the New Oxford American English Dictionary (OUP, 2016). Nonetheless, in effort to determine the greatest good for the greatestt number of people, when one discriminates first, then carries out acts against another member of society based on that distinction would be arbitrary. It would be difficult to substantiate ethically the decision by one person to choose someone else not deemed good enough solely on the basis of their being alive. I contend that unless the member to be sacrificed living directly kills the other four or indirectly Causes their deaths, choosing one not involuntary to sacrifice may be misguided. Moreover, distinguishing a subgroup from this group is possible: A group can be 3,4, or 5 members before or after; this group cannot change. There is something not quite right about the violation of individual rights which in the author's view, renders it to be unsuitable as an influential basis for decision-making in selecting criteria for allocation. Therefore, a deontological lens the author believes would be more appropriate for the tasks involved in resource allocation. Deontological frameworks are metacognitive in that they consider not only products like consequentialists, but duties or obligations to perform processes as well.

Allocation of resources in limited supply requires there be priority assignment of recipients. When considering potential criteria for use in determining prioritization, there are a variety of frameworks from within which the ultimate decision can be made. We already briefly discussed ethical perspectives from both deontological and consequential standpoints and some of the problems with consequentialist grounds. In addition to these frameworks, there are personological variables of the potential patients being considered for resource allocation. Personological variables may be understood as characteristics or properties that are unique to a particular individual or group (Martella, 2014). I would elaborate on this definition by adding that it is the set of characteristics or properties, not singular ones.

At first glance, some may argue that personological variables would be counterintuitive as a legitimate consideration given the context of objectivity and rationality the allocation of resources entails. Objectivity and rationality are crucial to ensure the most efficient use of resources. Unfortunately, though, because no two people 
have an identical set of characteristics, the characteristics must depend on and be unique to the person or group. However, if that is the case, then how could they be genuinely considered objective criteria?

Another contention might begin with the following: that the set of personological variables cannot be empty. For obvious reasons, without a single personological variable, there could be no allocation of resources possible. Additionally, since properties are contingent on the prior existence of something of substance (E1d5), by virtue of their existence, all things of substance can be said to necessarily have at least one property. However, depending on one's metaphysical perspective on substances and properties (i.e., monism versus pluralism), there may be a slight variation in framing the problem: Are people substances or properties?

Table 1.

Metaphysical Person-Perspective: Are People properties or subs tances?

\begin{tabular}{lcc}
\hline & \multicolumn{2}{c}{ Property } \\
\cline { 2 - 3 } Substance & Monism $^{\mathrm{a}}$ & Pluralism \\
\hline Monism & Pure Monism & 3 \\
Pluralism & & Pure \\
& Irreconcilable & Pluralism \\
\hline
\end{tabular}

\section{Substances or Properties?}

It may seem obvious to the reader or rhetorical, but the following question is sincere: Are people considered substance or property? This question is relevant to resource allocation and criteria selection because discrimination that allows distribution of resources is contingent on some properties being shared while others are not. Concerning our question regarding substance be versus property, although it may prove challenging to question the claim that, regardless of one's belief system, there exists more than one person, it is not impossible.

An individual who may argue that there exists no more than one person of whom one can be certain (i.e., Cogito Ergo Sum) may hold a monistic belief system with regard to person as one substance. However, for those who allow for the existence of more than one person, there would be a need to distinguish whether these people are multiple properties of one substance or multiple substances altogether.

Considering the assumption of persons as substance, it would be unreasonable to conclude that multiple substances exist but only one property be possible of them; properties or characteristics are perceived of substances so perceiving only one property 
no matter the number of "distinct" substances (i.e., persons) there may be would make it impossible to determine more than one substance or how many more there are. Thus, substance pluralism with property monism can be excluded as a potential framework because it would serve no purpose for criteria selection or allocation. Additionally, the converse of substance monism with property pluralism is equally moot. If there are different properties for one substance, then using properties as criteria for allocation end up making no difference since person as substance is singular and would receive the resource.

As for pure monism as a lens with people as substance, again without the ability to perceive more than one property or characteristic, there is no use for it in criteria selection or allocation. So, it appears as though persons as a single substance may not be the best choice for a primary factor with which to begin given the inability to discriminate regardless of the number of properties. We can claim with certainty that if people are substance, then there must be multiple properties to be of use as criteria for decisionmaking. Additionally, if people are property, then there must be more than one substance to be of use for resource allocation. Therefore, the only remaining tenable option is for genuine pluralism. Pure pluralism as substance pluralism and property pluralism may be most suitable as a guide for criteria selection and decision-making in transplant resource allocation because recipients can be discerned based on properties or characteristics, then prioritized accordingly.

The number of characteristics comprising any set is inversely proportional to the amount of potential candidates. That is, as the number of characteristics or properties comprising the set increases, the potential candidates decrease in number. Given the task of allocating limited resources, it then seems that there ought to exist an optimal number of properties or characteristics that allow there to be just enough as not to exceed the resources. It cannot be emphasized enough that caution must be exercised in selecting the characteristics because either choosing the wrong quantity of characteristics, or the incorrect qualitative properties could be disastrous. The only situation worse than having too few resources available to meet demand would be to underutilize the available resources. Resources would not only be inefficient, but wasteful due to inefficiency.

What the author finds somewhat troubling is that selecting the characteristics to result in an optimal number of candidates to minimize waste or overextension of resources then becomes dependent on the available resources, which will influence which sets of characteristics will be possible. So, instead of independent, objective criteria being chosen, the converse of what we intend, viz., to use an independent set of characteristics to determine who should get the available resources, is ultimately what occurs: the 
available resources will be used in order to determine the optimal set of characteristics to allow for supply to meet demand that candidates should have to ensure efficient use. In other words, optimal is in reference not to patient outcome, time to recovery, health status, improved quality of living, or likelihood of recidivism; it concerns primarily the resources available meet the demand instead of be wasted or overextended disappointing patients who got high hopes. Therefore, efforts to justly distribute resources most efficiently wind up dependent on availability, and availability determines optimization, which in turn dictates which sets of properties or characteristics as personological variables produce number of candidates match available resources even if the properties are not necessarily the most relevant, reliable, or accurate of potential patient outcomes. The question becomes, along with deontological ethics as a context and pluralistic metaphysical viewpoint, whether efficient use of resources (i.e., not wasting) takes precedence over the outcome of the potential patient. Is it better for transplant board or decision-makers to allocate the ten organs available once choice of properties that produce exactly ten recipients is determined, or consider personological variables that optimize outcomes at the expense of potentially wasting or giving false hope to those who will not get one?

Despite the difficult nature of the previous question, the author contends that how efficiency is understood is critical. Assuming the opposite as worst-case scenario, which outcome is best: using the set of properties that results in consumption of the exact number of transplant resources without having leftovers, but have half survive only, or select the set of properties that have the highest chance of the patients chosen surviving nonetheless those who don't qualify will unfortunately not survive with the transplant? In both scenarios, supposing equal numbers of people survive as do not survive: Is efficiency using criteria to optimize use so all ten organs are transplanted but have 5 go to waste by failing the patient, or finding criteria that maximize potential outcome of use but end up only using 5 transplants all of whom survive while the other 5 organs don't get used?

The author argues that, while both maximizing uses of resources available in the former case is reasonable as efficiency, as well as maximizing outcomes for efficiency in the latter case, efficiency as maximization of chances at successful outcomes would be most appropriate as an approach to efficiency. Framing efficiency as the maximization of chances of successful outcomes comprises maximizing use of resources as well as maximizing potential success of recipients. According to the hypothetical example used, a multitiered system incorporating both interpretations of efficiency that results in maximizing chances of success would ensure that the optimal criteria producing the five most likely candidates to be successful recipients are allocated organs and that the remaining five organs be allocated despite recipients being less likely to survive; chances, 
no matter how unlikely they may be at survival, will always be higher for candidates who receive organs than those who do not receive them.

To frame for maximization of chances, the author suggests personological variables. Though they may be peculiar to the individual, these characteristics relate to the person and could significantly affect the outcome of procedures utilizing allocated healthcare resources. It is for this reason that the author claims when a resource to be allocated is an organ for life-saving transplantation, among the most crucial personological factors that ought to be considered as a relevant criterion is the substance use history of the potential recipient. This factor as a theme will be discussed.

\section{Substance use history}

Substance use history of a candidate would be regarded as an appropriate personological variable for consideration because of the existence of the potential for continued use or relapse. The occurrence of relapse is relevant because it undermines the outcome through the return to use of licit and illicit substances, which may compromise the success of the organ graft either directly or indirectly. Directly compromising the outcome would be the case in which a liver transplant recipient resumes the consumption of alcohol that damages the organ received. Conversely, indirectly jeopardizing the result of a transplant would be the case of marijuana usage that leads to cognitive and functional impairment with poor decision-making (Volkow, Baler, Compton, \& Weiss, 2014).x

The paradigm for poor choices related to both cognitive and functional impairment caused by substance use would be the case in which one decides to operate a vehicle while under the influence (OUI). A meta-analysis has shown OUI to result in a twofold increase in the likelihood of having a collision in which serious injuries or death are a consequence (Asbridge, Hayden, \& Cartwright, 2012). Given the gravity of the potential consequences that directly or indirectly compromise organ transplant outcomes resulting from relapse and related to the personological variable of substance use history, the choice of approach to assessment criteria and prioritization for healthcare resource allocation is paramount. Since the seriousness of the consequences just discussed appears to be related to action and a lack of responsibility on the part of the substance users as demonstrated by engaging in harmful or reckless behavior, favorable approach options should address this aspect in their criteria. One such choice of approach promulgated that does incorporate aspects of action and responsibility is that of lower priority setting, which is the focus of the present article.

In support of the position of lower priority setting as an approach to healthcare resource allocation, Moss \& Siegler (1991) argued that organ transplant and other 
healthcare resources should not be provided on an equal basis to individuals who have failed to share responsibility for their health conditions. As it pertains to the present paper, such a failure on the part of people refers to the development of diseases or conditions that result from, or are associated with, responsibility related to action or behaviors concerning substance usage. The argument in support of lower priority setting is premised on the assumption that people with a health issue related to substance use and addiction have not done enough on their own (i.e., successfully stopped using substances) in addressing their medical condition. However, medical conditions that require organ resource allocation do not necessarily result from the decision to use substances. Moreover, in those cases that do result from illicit substance abuse, research has shown that there exists a genetic predisposittion to addiction that contributed to addictive behaviors (Nestler, Genes and Addiction).

Although contribution to one's health is required to merit equal consideration and avoid a lower priority setting, holding people accountable or penalizing them for their genetics to which they are often likely to succumb is tantamount to blaming them for that over which they have little to no control. Merely needing an organ transplant because of substance abuse history will not suffice since many are disadvantaged genetically. Thus, there may be no way to justify a lower priority setting even for those who require organ transplantation because of their own actions. In this paper, we consider whether lower priority may be substantiated. Ultimately, it is concluded that the lower priority setting denies potential recipients an equal opportunity to receive organ donations based on an argument that is hugely problematic.

The failure to share one's responsibility may be interpreted as a relative inequality that could be useful in the prioritization of patients in need of a healthcare resource. Such an inequality may substantiate "unequal treatment in proportion to the relevant inequality" according to Aristotle's formal principle of justice (Golan, 2010). Concerning this position, the unequal treatment could be that those with substance use histories would be given lower priority than the other potential resource recipients to whom the organs would be allocated.

\section{The Problematic Position in Support of Lower Priority Setting}

Despite the inherent problems associated with the lower priority setting, it aims to address the healthcare resource demands in society in an efficient manner. In an article by Golan (2010), it was suggested that because healthcare is a social good that cannot possibly address the needs of all people due to limits in quantity, a determination of relevant inequalities as criteria should be considered from the perspective of a utopian society in which resources were unlimited. The rationale for such a conception is that the criteria in a utopian society used for the resolution of ethical issues involving resource 
allocation would provide insight into how the problem facing the real world should be approached at a minimum. What is most appealing about a utopian thought experiment for healthcare resource allocation is the fact that a satisfactory resolution would be entirely independent of the quantities of societal resources available.

Society's approach to the distribution of healthcare resources should be both sensitive and specific about the demands of its constituent members' health needs. In this respect, which is analogous to and borrowed from the epidemiological meaning of the terms according to Boslaugh (2015), efficiently addressing a society's needs, whether utopian or otherwise, would mean that resources ought to be allocated in a sensitive manner such that only those who need resources because they stand to benefit from them receive them. Moreover, allocation in a particular manner should result in those who do not need them not receiving them. It would also be rational for resources to be appropriately allocated only after the needs, risks, and benefits among other things, have been adequately considered.

Many procedures would qualify as examples of efficiently prioritizing patients and healthcare resources within a society. Regardless which procedure a society may select, in one form or another, there must be some protocol for sequencing both resources and patients involved. The author suggests that such sequencing may be understood conceptually through a principle of well-ordering (Cunningham, 2012). When applied to society, because well-ordering implies that there is always a least element, it could aid in the determination of needs of, risks to, and benefits for members with health conditions. In this manner, once the decision is made, it then becomes possible to arrange cases according to most need, least risk, and most significant benefit so as to calculate an overall priority that facilitates the distribution of healthcare resources.

The concept of well-ordering has the potential to be most useful in patient cases being prioritized when done iteratively in series. Although well-ordering may be helpful, prioritization also relies on the consideration of needs, risks, and benefits that are associated with organ transplantation, which are presumed to have already been established in candidates by the time they are being evaluated for the procedure. Nevertheless, other factors such as substance use history must be considered in order to carry out the procedure of prioritization completely. Factors like substance usage potentially create difficulties in the process of prioritization and relate to deciding how best to frame the socio-philosophical problem concerning organ transplantation and healthcare resource allocation.

\section{Framing the Problem of Resource Allocation}


Prioritization and healthcare resource allocation may be framed in a variety of ways. For instance, it could be framed from the perspective of patients' rights, which according to Gillon (1985), may be considered "justified claims that require action or restraint from others (p. 1890)." Nevertheless, if it is granted that one does have rights, then through the requirement of others to act or refrain from acting it may be inferred that others must have a duty or obligation (i.e., deontology). Also, the perspective of right conduct (i.e., morality) may serve as a reasonable framework. That notwithstanding, from the standpoint of what the patient can reasonably look forward to or expect, prospect theory may also be a viable choice to consider. Moreover, the issue could be addressed from the viewpoint of dessert under the rubric of justice. Regardless of the perspective from which the problem may be framed, whether from one of rights, deontology, morality, prospect, or justice, there always exists some degree of overlap.

It seems as though, given the interrelatedness between the previously mentioned perspectives, a reasonable foundation for any issue of this nature, as Golan (2010) indicates, would be Aristotle's formal principle of Justice: "Equals should be treated equally; Unequals should be treated unequally, in proportion to the relevant inequality." That notwithstanding, it would be difficult to consider substance users as "unequal" and their self-injurious behavior as the relative inequality for a determination of how to treat them in proportion to such inequities due to the involvement of multiple factors regardless the perspective from which the process of assessment begins.

\section{Approaching from A Standpoint of Logical Consistency}

Of the views available from which to address the present issue, there is an integral aspect common to all: logical consistency. The reason that logical consistency is fundamental is that regardless the framework from within which one chooses to address the issue of healthcare resource allocation and lower priority setting, principles peculiar to each one must be internally consistent. Furthermore, from such internal consistency, once overlap and interrelatedness are taken into account, consistency is the sort of thing that may be capable of unifying the strengths of one framework with those of the others. Thus, by extrapolating and applying aspects from one approach to other perspectives, such an integrated approach may prove fruitful to progressing toward a solution.

To understand how one viewpoint leads to others, suppose duties or moral obligations are the starting framework. It is acknowledged that duties, or moral obligations, are the domain of deontology (Gillon, 1985). The question that arises when deontological aspects are considered, however, concerns what it is about the duty or obligation that allows it to be situated within the province of morality, or "right conduct." In other words, are duties or obligations moral due to some constitutional principle of justice that inheres within them, or is the morality a consequence of others having 
acknowledged one's justified claims (i.e., rights)? Alternatively, perhaps it is the prospect of one's justified claims being legitimized by the duty that others feel that makes fulfillment a moral obligation. Nonetheless, if this were the case, then the "right-conduct" would be about the expectations being based on getting what one deserves, which would relegate the issue to the realm of justice. It is evident that they are all both valid and interrelated points that should be explored. However, they are exceedingly complex and may not necessarily be required to provide answers to address the problem.

On the other hand, starting with a moral question within the framework of rights, the issue of healthcare resource allocation and substance users concerns whether and how much one's role in choosing to engage in self-injurious behavior resulting in a health condition should be considered in determining the individual's priority (Golan, 2010). That is, whether one has the right to receive treatment when one is the cause for the treatment to be needed. Also, what about the provision of treatment in instances of selfinflicted harm render it "right conduct?" Moreover, the moral standpoint entails questions concerning deontological aspects of the issue because granting certain moral rights may be understood to imply the existence of duties or obligations (Gillon, 1985). In other words, the duties and obligations would satisfy the "right conduct" clause to be considered moral obligations. Furthermore, to employ a moral approach to the problem of healthcare resource allocation entails some notion of justice as well. That is, to say, getting what one deserves in the case of people with self-inflicted conditions, such as those from the use of licit and illicit substances, introduces into the equation how important what one does is in what another ought to do for them and why this may be the case.

It has been shown that there are many aspects of the problem from which the evaluation may begin. Although vital to consider regarding the topic of substance users and organ allocation, because any of the individual perspectives is comprised of more than consistency each may be sufficient to frame the issue while not being necessary to do so. For this reason, a somewhat different approach has been herein attempted. Thus, this work is not approaching resource allocation from the traditional frameworks arguing on the basis of consequences or obligations for their own sake; it will address equitable resource allocation and substance users from an integral framework of logical consistency, as the fundamental guiding principle. The employment of such a framework will allow the determination of whether this indeed is an issue concerning substance use as a relevant inequality.

\section{Equitable Allocation and Logical Consistency}

The process of equitable healthcare resource allocation is very complicated. As such, to approach the problem solely from any single framework would be insufficient at 
best. In other words, the fair allocation of healthcare resources should not just concern substance users' rights, nor should it be about the principle of justice associated with getting what one deserves. Righteousness should not be appealed to by itself because the allocation of organs as healthcare resources for substance users is not only about engaging in "right-conduct" on the part of healthcare personnel on transplant committees. For that matter, allocation of organs as healthcare resources may not just be about the duty or obligation imposed on healthcare staff or society. Moreover, distribution of these resources can no more be about the prospect of receiving an organ and having expectations fall short than it could be about judgment and bias dictating whether expectations substance users have for organ transplant should be met.

To address the problem of organ distribution as a healthcare resource allocation concerning substance users, an integral approach was selected. Adopting an integral perspective consists of maintaining logical consistency regardless of the framework from which the issue is viewed. Logical consistency is paramount to resolving the issue through the use of casuistry in its non-pejorative sense. Through the use of casuistry, logical consistency will govern the hypothetical case comprising a substance user in need of an organ transplant who contends with a non-user for the same organ graft resource. The guidance afforded by logical consistency in the extrapolation and application of moral principles or rules on which one may generally rely from any of the perspectives to new circumstances in the same case, or another case altogether facilitates the process of resolution. That is, consistency will allow for commonalities to reveal themselves and convergent thinking to assist in finding the core of importance. It is in this respect that the approach may be understood as integral.

It is a goal of this paper to demonstrate that fair organ transplant resource allocation should be adhered to according to the argument of the lower prioritists. If successful in proving the goal, then either the position of lower priority is directly challenged with the result of them being proven wrong for having excluded substance users, or their position is upheld, but leave no alternative but to accommodate substance users and prove the author is right. It will be interesting to ponder of whether they would abandon that position in exchange for another more exclusive one precluding substance users once again proving that the author was correct. Alternatively, would the prioritists hold firm in their resolve, yet allow their position to be more inclusive so that the disparaged substance users may be given full consideration thereby proving that they were incorrect? The challenge begins by looking at the role of intention and substance use.

\section{The Role of Intention}


There may be no straightforward way to demarcate issues of concern for medical justice from those of societal justice. That is, the notion of dessert with respect to medical treatment may implicate social responsibilities or obligations. So, if a substance user has the right to organ allocation for transplantation, that he or she may not be able to afford it themselves when society can afford it, could mean that society has a duty to subsidize the graft procedure. Nonetheless, if it may be demonstrated that the individual has relinquished or otherwise lost membership, then that obligation no longer exists. The problem is what it would take to convincingly prove someone has behaved in such a way as to forfeit his or her membership. Furthermore, how would one show that engaging in such behavior was or was not a free choice?

The assumption that individuals behaving in a non-meritorious manner choose actions of their own volition may be seriously questioned. Volition as freedom of choice may seem apparent when, in fact, it results from a combination of genetics and environmental circumstances. Nevertheless, instead of subjecting free will to the traditional approach of a direct challenge, it may prove more beneficial to do so via testing another implicit assumption freedom entails from the perspective of self-inflicted injuries: that they were intentional.

It is assumed that self-inflicted harm as a free choice is the intention. However, self-inflicted injury as free choice is not necessarily the intention of an individual. That intention may have existed related to harming oneself does not necessarily correlate with outcomes of actions either. For instance, suppose that an individual was trying to save someone's life. The intention to save someone's life was made known immediately before acting. While it is possible that this person may have intended to save another's life, in the act of performing CPR on this individual, he or she breaks a rib that punctures the heart. As a result of the fractured bone, the person subsequently dies. It may be gleaned from the events in this situation that what was intended was not the outcome even though the intention was carried out through the action. Every action was backed by intention in the hypothetical scenario, yet the outcome was not what was intended. At what point does intention become the warrant that substantiates the claim of action as a causal link in the chain of events leading to the outcome?

Aside from the outcome of harm or death being distinct from the act of selfinfliction that preceded it being beyond one's control, just because one may be responsible for the injury sustained in no way necessarily implies that they desired or intended it to be. Some distinctions may be made between intention, action, causation, and outcomes; they are all interrelated, but they do not necessarily implicate one another. If one intends to do something, then he or she intended when they do that something; but it is not the case that if he or she does something, then he or she intended to do it. The 
action performed, and the intention itself are separate from one another. While they may coincide with one another, the expressed intention to act and the commission of the act intended itself do not necessarily coordinate with one another. For instance, the act of swinging a stick blindfolded with the intention of breaking a pinata may result in the commission of the act of hitting someone instead; thus, what was intended (i.e., hitting a pinata) was not what was committed (i.e., hitting a person). In this manner, the intention and commission of acts are seen to be separate.

It is possible to be responsible for doing something one intended to do that was a proximate cause of a result that one did not intend. The intention may not be translated into the occurrence of an outcome regardless of the proximity to it. Concerning selfinjurious behavior, self-inflicted harm, or death that occurs is an outcome on which one's intentions may have no influence; one's intention only influences one's action and behavior but neither consequences nor outcomes.

If one has the intention of doing something and does it, then that something as the action is the one that was intended to be done. Important to inquire is whether one may intend to do something that one knows there is no plausible way to do it. In other words, could a person with no legs truly intend to play hopscotch? Alternatively, is it the case that intentions must infer possibility, if even remote? That one claims to intend on walking to the store necessitates the ability to walk. Otherwise, to speak of intentions are meaningless. The role of intention should be to establish what is possible. In other words, intention does not mean that something is the case, but only that something can be the case.

To actually qualify as intentions, what is claimed as such must allow one to infer that what is intended is indeed within the realm of possibility of the one claiming it. There are at least two perspectives of the situation concerning possibility. In one perspective, an ability exists, but the limits of the ability are insufficient to meet the expectation. In the other, there is an absence of ability. In the former case, if there is a five-foot-tall brick wall that someone claims to intend jumping over yet he or she can only jump four feet, despite having the ability perform the act, one cannot honestly claim this intention. Although jumping over a wall is possible, the ability is insufficient to meet the demand. In the latter case, a person who claims to have the intention of playing hopscotch must have legs otherwise to do so is not within the realm of possibility for him or her either. Therefore, whether the ability exists determines what is possible. That notwithstanding, in the presence of ability, what is possible is contingent on demands meeting one's capabilities.

Another aspect of intention implicit in possibility is to control. While having legs is a requisite to engage in any activity that involves them, the possession of legs alone is insufficient; one needs to be able to go from not using legs one has to use them for a 
particular purpose. The ability to use one's legs requires control of them. For instance, both the lower limb double-amputee without legs (therefore, without control of legs) and the person with paraplegia who has legs over which there is no control, are unable to intend to engage in activities such as hopscotch honestly. Moreover, if it is true that the intention to play hopscotch requires possibility, the possession of legs, and control of what is in one's possession, then intentions claimed also require the first-person perspective. That is, it is never the case, nor is it possible, that one may claim intentions in any other person-perspective legitimately. In other words, someone may intend something, or someone may intend for something. Nevertheless, it is the someone who from their perspective doing the intending such that he or she may not intend anything from an alternate view that is beyond his or her control. Thus, the following may be concluded: (1) intention requires possibility, (2) intention seems to concern the ability for actions to occur, (3) intention requires possession of means needed for the ability, (4) intention implies control of what is possessed, and (5) intention requires the first-person perspective and performance by the one claiming the intention. Based on propositions 1 through 5, intentions, therefore, imply actions of some sort, but do not necessarily imply consequences.

Although the act of killing, for instance, is understood to be a verb in that to kill is thought of as something that is done, the author contends that it is not; unlike to walk or to otherwise do, to kill is not a pure act. Killing as a verbal noun does not refer to a valid action - it is in reference to a consequence. Because the occurrence of the gerundial killing is contingent on the completion of something else prior, its meaning is conveyed in a conditional sense when used. Thus, killing does not have the status of an action.

Nevertheless, if a pure act such as to stab the heart with a knife may be established as a particular action that causes one specific consequence like another's death, then to stab the heart with a knife may be equated with a pseudo-action of killing. In this manner, killing may be considered a pseudo-action resulting from a real act plus the consequence of that action. Therefore, when a pseudo-act such as killing may be equated with a true action, the commission of a specific act one intended that causes a particular result cannot occur without one being held liable for the specific consequence as an outcome.

The true action/pseudo-action dichotomy is relevant to the present paper because "getting cancer," "having a heart attack," or "killing oneself" all are pseudo-actions. In order for them to be considered true actions, there must be a true action plus a specific consequence of that action. For example, if killing oneself is to be equated with an intentional act and not a consequence, then it requires a true action plus the respective result of that action being losing one's life to occur reliably. In the case of blaming substance users for getting liver failure that requires organ transplantation, consuming 
alcohol would need to be the true action, and liver disease would need to be the consequence that always occurs. Together, the true action and the specific consequence result in getting liver failure, which could then be justified as a true action. Nevertheless, this does not mean that one intended to get liver failure.

Although not an action, one's intention may be transitively related to the outcome only until the point of completion of the action serving as a temporal precursor but not necessarily cause. This finding is vital to the present paper and places substance users' actions in the context of behavior up to the point of generic cause without the outcome being directly consequential. It is argued that for the substance users needing organ transplantation, the health condition that they developed after engaging in their addiction behavior may have occurred without their conduct; therefore, there is no way to establish it as a direct consequence even if they intended to behave the same way acutely aware of the possible sequelae.

\section{Argument Against Assigning Lower Prioritization}

The argument for, and approach to, prioritization seems to be quite problematic. For example, if we begin by assuming someone needs a liver transplant because of alcoholic liver disease (ALD), which is a result of substance addiction, then we can infer a few things. Given there is evidence to suggest that there may be a heritable predisposition to substance dependence (Sora, 2014), it is reasonable for someone so predisposed to be more likely to become addicted and have difficulty to stopping. If we compare this former user to a non-user who needs a liver transplant, then they are probably vying for the same available organ. The lower prioritists would claim that, because of ALD, the former addict has not done enough to address his or her health (i.e., tried hard enough to stop drinking) to deserve equal priority to the nonuser. Nonetheless, this is not necessarily the case.

Since having ALD as a result of substance addiction does not reveal the steps taken to stop drinking, there is no way to know whether this person did or did not do enough; a lack of success does not imply a lack of effort. Furthermore, the non-user who presumably needs a liver transplant unrelated to actions that involved taking substances has not done anything to address his health issue because he or she could not; there is no way to know whether it was within his or her control so they knew nothing that they could have done would have been directed toward affecting the development of the disease condition.

Since substance use and addiction has been shown in twin studies to be influenced by genetics and environmental factors beyond one's control, if predisposed to use or abuse, then actions may be insufficient to avoid beginning or discontinuing a substance 
use habit. Thus, it is possible that no amount of action would override an individual's propensity to become addicted or successfully quitting usage. In other words, either an addict could do enough or could not do enough to address their health condition and make themselves quit.

If a substance user could do enough, even if a liver transplant were still needed, then more would be done to quit using the substance than a non-user who needed a liver transplant; and if a substance user could not do enough, then either he or she has not done anything because they cannot, or has done everything trying incessantly to no avail. Important to note is that if an individual has not done anything, then he or she would be doing as much as the non-user (i.e., nothing), yet if someone has done everything trying incessantly to no avail, then he or she would be doing more than a nonuser. The addict either would do the same as the non-user did by doing nothing, or he or she would do more than the non-user did regardless whether the addict applying for a livercould do enough to quit successfully, or could not do enough and failed miserably. Therefore, if priority is being based on "doing enough," and we have just seen that non-users in need of a transplant have not done anything, then addicts either should get at the least the same priority as non-addicts, or they should get higher priority. That is, the potential genetic influence implies that the substance user could give more effort by doing more than non-users yet find themselves in the same predicament. In this respect, this argument not only refutes that of lower priority, it is asserting that substance users should not be demoted to lower priority, but that, according to its condition of moral responsibility obliging one to do enough, the priority could justifiably be given to those with substance use histories, as the author has just presented.

\section{Conclusion}

While lower priority setting may be substantiated in some instances for individuals if their substance use may be proven not to be due to factors beyond one's control, the argument in support of prioritization based on personal responsibility to address the problem of equitable distribution in healthcare resource allocation is untenable. If it is true as research has suggested, the existence of heritable factors, or those otherwise beyond one's control, are involved in substance use or abuse, then substance users may not be held responsible for not doing enough to address their health. Furthermore, not sharing responsibility by failing to do enough (i.e., the negation of action) does not logically imply that the lack of substance users' success results from a lack of effort. Also, given that a lack of success cannot be reasonably inferred from a lack of success, as well as the existence of factors otherwise beyond one's control such as genetics and environment to a degree, substance users should be given equal or higher 
priority than non-users. Those with substance use histories should be given equal or higher priority because either they may have done as much as non-users to address their health, or they may have done more than non-users to address their health regardless of their level of success.

It was useful to ponder the way a utopian society would resolve ethical issues related to healthcare resource allocation because there was no need to take into account the quantity of available resources. Such limitless supplies reinforced an approach to distributing resources that was both sensitive and specific. That is, concerning the demands of the members of any society, to efficiently meet the needs resources ought to be disbursed only to people who have a need and may benefit from receipt. Moreover, a specific approach to allocation would ensure that those without a need or could not benefit from receiving them did not get them.

To facilitate the allocation of resources, prioritization must occur beforehand. Priority setting as sequencing was likened to a principle of well-ordering in which there was always a least or lowest element. As applied to society, well-ordering would help to establish who would receive resources first based on needs, risks, and benefits. Setting the order of priority depends on other factors, such as those considered personological. Personological factors like substance use, however, could lead to encountering obstacles in setting the order.

The challenges faced are related to figuring out the best manner in which to frame the problem of the distribution of organs as healthcare resources. Nonetheless, irrespective of the standpoint from which the problem is framed, whether rights, deontology, morality, prospect theory, or that of justice, there is always a certain amount of interdependence and overlap. Although Aristotle's Formal Principle of Justice has been chosen by some to be a starting point for issues relating to ethics, framing substance users as the unequal and their substance use as the self-inflicted harm that is the relative inequality may not be the most appropriate approach. Interpreting substance usage as the relative inequality to determine the proportional treatment according to such inequities invites many difficult challenges no matter the perspective ultimately selected.

As opposed to relying on Aristotle's principle, arguing on the basis of consequences or obligations, or any of the other traditional frameworks for approach, it was suggested that a principle of logical consistency guide the approach. The suggestion was based on the exceedingly complex nature of the other approaches, any of which may have been sufficient without being necessary. The complexity refers to the other aspects of the particular framework beyond the foundational element serving as the philosophical binder. As a philosophical binder, that foundational aspect was logical consistency. Each framework was logically consistent as a distinct philosophy, yet it was 
shown that any one of the perspectives includes aspects that can be derived from another of them. Such interrelatedness among the perspectives implied the existence of a commonality. Moreover, as the commonality, it was logical consistency that was thought to allow capitalization on the strengths of the perspectives while also compensating for the individual weaknesses.

Through drawing on strengths to overcome shortcomings and appealing to the fundamentals, the approach was deemed integral. The notion of a desert as it pertains to substance users receiving organs as healthcare resources to be distributed for medical treatment may require the existence of social responsibilities or obligations in society. However, the duties or responsibilities that society may have to substance users could only exist as far as they maintain membership. According to some, substance users freely choose to engage in behavior that causes the conditions for which organ transplantation is required. From the viewpoint of self-injurious behaviors, implicit in the presumption of freedom to harm oneself is that an act or behavior is intentional. Nonetheless, it was pointed out that the outcome of injury or death subsequent to a self-inflicted injury is distinct from the self-injurious behavior that preceded it. Furthermore, although the result itself was shown to be beyond one's control, even when an individual may be held responsible for the action the lead to an injury sustained, it was not necessarily the case that he or she intended the action or the outcome. It was, therefore, concluded that while they may coincide at times, intention and commission of acts were, in fact, distinct from one another. In addition, actions committed may or may not be causes, but as causes, they may or may not be associated with specific outcomes. That is, intention, action, causation, and outcome as the chain of events are interrelated yet independent.

It was inquired as to whether one may really intend to do something that one knows there is no plausible way to do it. The argument that was provided led to the conclusion that one's claiming to have the intention of walking necessitates his or her ability to do so to avoid meaninglessness. Intention has the primary purpose of either establishing what is possible or allowing the inference of what lies within the realm of possibility of the individual claiming to have the intention. It is possible to be responsible for doing something one intended to do that was a proximate cause of a result that one did not intend. The intention may not be translated into the occurrence of an outcome regardless of the proximity to it. Concerning self-injurious behavior, self-inflicted harm, or death, that it occurs is an outcome on which one's intentions may have no influence; one's intention may only affect his or her actions or behavior. Nevertheless, neither consequences nor outcomes as a result of actions or behavior may be influenced by one's intentions. Therefore, it is not that intention means something is the case; intention only indicates that it is possible for something to be the case. 


\section{References}

Asbridge, M., Hayden, J. A., \& Cartwright, J. L. (2012). Acute cannabis consumption and motor vehicle collision risk: systematic review of observational studies and metaanalysis. BMJ e536, 344 SRC - GoogleScholar.

Alexander, L., \& Moore, M. (2016). Deontological Ethics. In E. N. Zalta (Ed.), The Stanford Encyclopedia of Philosophy (Winter 2016). Metaphysics Research Lab, Stanford University. $\quad$ Retrieved from https://plato.stanford.edu/archives/win2016/entries/ethics-deontological/

ATF. (2008). American Transplant Foundation: Facts and Myths about Transplants (Vol. 2008 SRC - GoogleScholar).

Boslaugh, S. Statistics in a Nutshell, 2nd Edition, 2nd ed. Place of publication not identified: O'Reilly Media, Inc, 2012. Kindle 6.

Dew, M. A., DiMartini, A. F., Steel, J., Dabbs, A. D. V., Myaskovsky, L., Unruh, M., \& Greenhouse, J. (2008). Meta-analysis of risk for relapse to substance use after transplantation of the liver or other solid organs. Liver Transplantation, 14(2), 159172.

Gillon, R., \& Gillon, R. (1985). Philosophical medical ethics. rights. British Medical Journal (Clinical Research Ed.), 290(6485), 1890-1891. doi:10.1136/bmj.290.6485.1890

Golan, O. (2010). The right to treatment for self-inflicted conditions I Journal of Medical Ethics. Retrieved from http://jme.bmj.com/content/36/11/683.long?utm_source=TrendMD\&utm_m edium=cpc\&utm_campaign=J_Med_Ethics_TrendMD-0

Jones, E. E., \& Nisbett, R. E. (1971). The Actor and The Observer: Divergent Perceptions of the Causes of Behavior, 79-94.

Lincoln, Y. S., \& Guba, E. G. (1985). Naturalistic inquiry. Beverly Hills, Calif.: Sage Publications.

Liu, L. U., Schiano, T. D., Lau, N., O'Rourke, M., Min, A. D., Sigal, S. H., . . Bodenheimer, H. C. (2003). Survival and Risk of Recidivism in Methadone-Dependent Patients Undergoing Liver Transplantation. American Journal of Transplantation, 3(10), 1273-1277. 
Luckerhoff, J., \& Guillemette, F. (2011). The conflicts between grounded theory requirements and institutional requirements for scientific research. The Qualitative Report, 16, 396-414.

Machi, L. A., \& McEvoy, B. T. (2016). The literature review: Six steps to success [Kindle 6].

Martella, R. C. (2014). Understanding and interpreting educational research [Kindle 6 Version] (1st ed.).

MOSS, A., \& SIEGLER, M. (1991). Should alcoholics compete equally for livertransplantation? Jama-Journal of the American Medical Association, 265(10), 12951298.

Ranney, D. N., Acker, W. B., Al-Holou, S. N., Ehrlichman, L., Lee, D. S., Lewin, S. A., . . Englesbe, M. J. (2009). Marijuana Use in Potential Liver Transplant Candidates. American Journal of Transplantation, 9(2), 280-285.

Sora, I. (2014). PL8GENETIC VULNERABILITY OF DRUG ADDICTION. Alcohol and Alcoholism, 49(suppl_1), i2-i2. doi:10.1093/alcalc/agu051.8

Volkow, N., Baler, R., Compton, W., \& Weiss, S. (2014). Adverse health effects of marijuana use. New England Journal of Medicine, 370(23), 2219-2227. doi:10.1056/NEJMra1402309

Wells, J. T., Said, A., Agni, R., Tome, S., Hughes, S., Dureja, P., \& Lucey, M. R. (2007). The impact of acute alcoholic hepatitis in the explanted recipient liver on outcome after liver transplantation. Liver Transplantation, 13(12), 1728-1735. 